\title{
LEARNING FROM THE MARS ROVER MISSION: SCIENTIFIC DISCOVERY, LEARNING AND MEMORY
}

\author{
Charlotte Linde
}

\begin{abstract}
Purpose

Knowledge management for space exploration is part of a multi-generational effort. Each mission builds on knowledge from prior missions, and learning is the first step in knowledge production. This paper uses the Mars Exploration Rover mission as a site to explore this process.

\section{Approach}

Observational study and analysis of the work of the MER science and engineering team during rover operations, to investigate how learning occurs, how it is recorded, and how these representations might be made available for subsequent missions.

\section{Findings}

Learning occurred in many areas: planning science strategy, using instruments within the constraints of the martian environment, the Deep Space Network, and the mission requirements; using software tools effectively; and running two teams on Mars time for three months. This learning is preserved in many ways. Primarily it resides in individual's memories. It is also encoded in stories, procedures, programming sequences, published reports, and lessons learned databases.
\end{abstract}

\section{Research implications}

Shows the earliest stages of knowledge creation in a scientific mission, and demonstrates that knowledge management must begin with an understanding of knowledge creation.

\section{Practical implications}

Shows that studying learning and knowledge creation suggests proactive ways to capture and use knowledge across multiple missions and generations.

Value

This paper provides a unique analysis of the learning process of a scientific space mission, relevant for knowledge management researchers and designers, as well as demonstrating in detail how new learning occurs in a learning organization.

\section{Recognizing and Preserving Learning}

This paper describes an aspect of knowledge management that is rarely discussed: the creation of new scientific, technical and organizational knowledge as part of an exploration mission, and the distribution of that knowledge within and across institutional boundaries. Knowledge management for space projects and institutions shares many of the same challenges as knowledge management for any other complex endeavor Personnel must be trained, policies must be implemented and documented, records and documents must be created and stored.

However, a scientific mission into space has several properties that add additional complexity to knowledge management requirements. First, the entire motivation for the mission is to develop new scientific knowledge. This knowledge results from learning by individuals, groups, and institutions. Much of the literature on institutional learning has focused on learning in the service of fundamental corporate goals, rather than on situations where the 
development of new knowledge is itself the goal. (See, for example, (Davenport and Prusak, 1988), chapter 3.)

An additional complexity is that space missions are commonly a collaboration between multiple institutions. In the case of the MER mission described here, the team included members from at least five NASA centers, with the Jet Propulsion Laboratory as the lead, as well as over 37 universities, institutes, and private corporations. This means that recognizing, preserving, organizing and disseminating new knowledge can not be confined within the boundaries of a single organization.

The most familiar form of knowledge captured in space knowledge management efforts is represented in the form of documents generated as part of the ongoing process of a mission: design documents, records of mission reviews, records generated during the mission, etc. However, there is also a great deal of retrospective activity attempts to recognize and preserve learning at the end of the project, or even more challenging, years later, from retiring experts. These post-project capture efforts most often consist of requirements for personnel to fill out computer-based forms in Lessons Learned systems and to deposit mandated records when departing. Somewhat less frequently, there is an attempt to capture learning by an interview process which results in written summaries or video records of stories told by departing experts.

In order for such efforts to result in useful knowledge consistently and effectively, it is important to ask what learning is in the context of space exploration, and how to recognize it. While so-called Lessons Learned databases are common, their underlying definition of a lesson is frequently not clearly specified. Most often, a lesson is taken to be the product of a mistake or a mishap: something which must be documented to assure that it will not happen again. Many such "lessons" are to be found in mishap reports by investigation boards, and many reporting databases have as their primary purpose the collection of data to be provided to such a board in case of a mishap. This is a negative view of learning as an unpleasant activity, as expressed in the colloquial phrase: "Have you learned your lesson yet?" This means that much more attention is paid to failed projects than to successful ones (MacCormack, 2004).

Yet in spite of mishaps at worst and reinventions of the wheel at best, much new and positive learning does occur As NASA prepares for a return to the moon after thirty years, more is known now, both technically and institutionally. Thus, rather than merely focusing on lessons learned in the narrow sense of documenting mistakes, there is a more inspiring question: how do people, projects, and institutions learn new things, how is this learning preserved and continued, and how is it possible to plan for such learning and preservation across multigenerational missions?

There are many types of learning in projects as large, complex, and lengthy as space exploration missions. At the highest level, there is mission-scale learning. This includes such issues as how to maintain support for a mission over many years, multiple elections, and changes of government commitments to the mission, and how to manage across changes in the requirements for how a government agency is legally mandated to work with contractors, and in the mandated system for management processes. (For example, (Johnson, 2002) describes changes in systems engineering models in American and European space programs.) At perhaps the most detailed level, there is learning about individual components and their behavior The behavior of the Space Shuttle's O-ring at low temperatures is perhaps the most 
notorious example, but any spacecraft design incorporates hundreds of thousands of such lessons learned about components and their interactions. At an intermediate level, there is the learning generated in the design process: inheritance of previous mission design concepts; development of new designs, materials, and processes; discovery by iterative design, etc.

In order to describe what learning looks like as it happens, this paper focuses on an intermediate level of learning that falls between the level of government policy and the level of widgets, looking at ongoing learning during mission operations, and the ways in which this learning is formally or informally incorporated into the memory of the institution performing the mission. It is difficult to capture or even recognize this kind of learning without special efforts. It may become part of the mandated or informal work procedures, incorporated into software code, or may simply become what "everybody knows." By the end of a mission, it is easy to forget that learning has taken place, since it has become obvious "common sense." Some learning may be captured but stored in disparate places so that it would be very difficult to reconstitute. Finally, without the intention and ability to recognize learning on the fly as learning, there is no reason to try to capture it systematically.

This paper first describes what such learning looks like, describes how such learning is currently captured (or not), and finally, discusses methods for discovering, capturing, and representing these more subtle forms of learning. In effect, the paper shows empirical examples of a few of the many types of learning processes which produce the data and information which knowledge management systems attempt to manage.

\section{RESEARCh SITE AND METHODS}

This paper is based on a study of the Mars Exploration Rover mission. At the time of writing (August 2005), the mission still continues, with both rovers in operation approximately 18 months after the mission's nominal duration.

NASA's Mars Exploration Rover Project consists of two rovers performing robotic geological fieldwork on two locations on the surface of Mars, searching for evidence of a history of past water activity The first rover, Spirit, landed on Mars on January 4, 2004, followed by the landing of the second rover, Opportunity, on January 25, 2004. The rovers were designed to recognize and maneuver around small obstacles on their way to observe features selected by scientists from images sent by the rovers. They carry an instrument package which includes a panoramic camera (Pancam), a stereo camera with 11 color filters and 2 filters for imaging the Sun, a miniature Thermal Emission Spectrometer (Mini-TES); a robotic arm with a microscopic imager to produce extreme close-up images of rocks, soils and particles; an alpha-particle spectrometer to detect elemental abundances of rocks and soils; a Mössbauer spectrometer to distinguish iron-bearing minerals; and a Rock Abrasion Tool (RAT) for brushing and grinding. Additionally, the rovers contain two monochromatic navigation cameras, four hazard avoidance cameras, and high and low gain antennae for data transfer to Earth. 


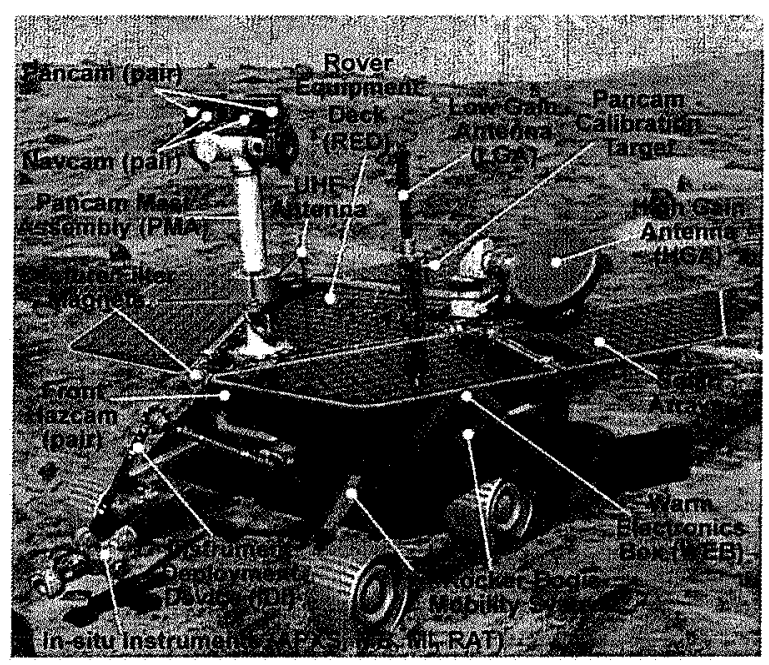

\section{The Rover's Instruments}

In addition to returning unprecedented data about Mars, the MER mission also has provided us with an opportunity to track how the science and engineering team learned, how this learning was recorded, or assimilated without specifically being marked as learning, and whether the representations of this learning are likely to be usefully available for subsequent missions. This paper is based on an observational study of the work of the MER science and engineering teams during the first six months of the mission, the most intensive period for these teams. Conducted by members of NASA Ames Research Center Work System Design and Evaluation team, the study included ethnographic observation, video and audio recording of the work of the science team, analysis of documents and technology use, and interviews with team members.

Grossly simplified, the work of the science operations team during the initial period consisted of receiving downlinked data from one of the rovers, assembling it into usable data products, analyzing it to determine what data to request next, planning the rover's activities on the next sol (martian day), and creating command sequences to uplink to the rover (See also (Wales et al., 2005) for a fuller discussion of the work of this team.)

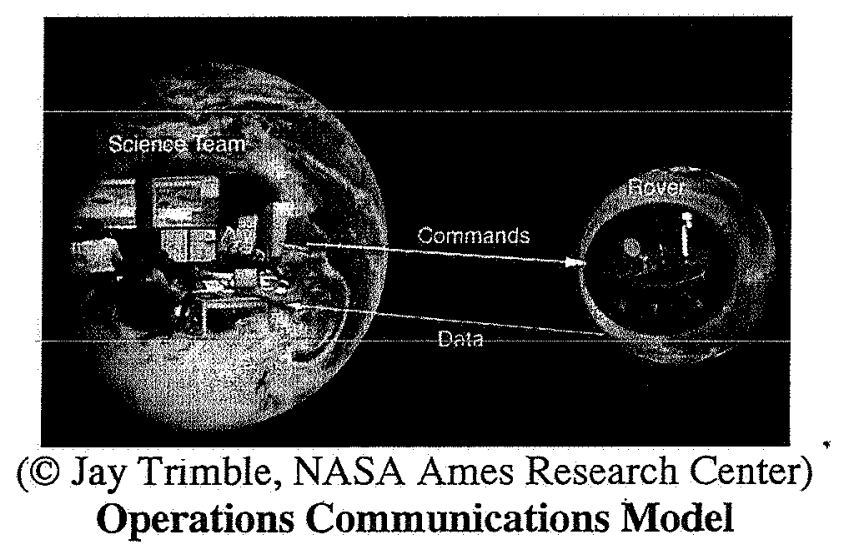

The science team was divided into five theme groups by scientific discipline: Atmospheric Sciences; Geology; Soils and Physical Properties; Geochemistry/Mineralogy; and Long Term Planning. Long Term Planning is a special group charged with developing a general plan for each sol, and ensuring continuity between sols, and tracking the progress of science and 
mission objectives. Discussion, learning, negotiation, and planning occurred in a series of formal meetings, punctuated by individual or ad hoc small group work.

\section{TYPES OF LEARNING}

There were many forms of learning and learning capture observed during the work of the science and operations team. Indeed, this paper describes a work in progress, since at the time of wiriting the mission still continues, over a year longer than the nominal 90 day duration of each rover's mission. Further, although several issues of Science have already appeared with initial findings from the Rovers, much of the work of scientific analysis, and assessment of design and operations decisions can be performed only after the conclusion of the mission. However, even before the end of the mission, it is possible to sketch some of the major areas of learning and knowledge preservation.

\section{Learning the Instruments}

A major type of learning we observed was the team coming to understand the potentials and limits of the instruments of the rover, and how to work with them to obtain the desired science return. The team had to strike a balance between the scientific desire for as many observations as possible, made at the highest resolution possible, and the practical restrictions imposed by available rover battery power, and bandwidth for downlink on the Deep Space Network (the "space internet" consisting of satellites orbiting Mars as well as three radio telescopes on Earth), which must transmit the data of many missions.

Let us consider in detail one example of many, observed three weeks after landing, a point in the mission when much new learning was happening. In an informal meeting, a group of scientists discussed possibilities of compressing data from the Microscopic Imager to overicome a potential downlink problem of too high a data volume for uncompressed images. The loptions were either to reduce the number of bits per pixel or to take fewer observations, or some combination of these strategies. The discussion included expertise offered by the writer of the data compression algorithm, who said that "One bit per pixel is far from adequate" while three bits cannot be distinguished from lossless compression. One of the scientists showed the results of a rapid experiment he has performed in the last twenty minutes, producing images at a low pixel rate. The chair of the Long Term Planning Theme Group suggested a compromise, and an immediate decision was made that a rate of three bits per pixel with five rather than seven slices was adequate for the current task, but that the decision might be revisited for observations on different soil.

This is an example of practical learning on the spot: learning how to use an instrument in new conditions within the changing constraints of the situation. It becomes an immediate part of the team's knowledge: a certain compression rate for this instrument will be adequate for certain scientific purposes, and thus can relieve some of the pressure of the volume of data to be downlinked.

The question may be raised: why call this learning? Isn't it a normal part of the scientific discovery process? There is certainly a great deal of overlap between the terms "discovery" and "learning" in science. However, it seems valuable to reserve the term "discovery" for scientific results: i.e. the MER mission has discovered evidence of the past existence of liquid water on Mars. "Learning" then can be used to describe the ongoing work of scientists as they develop the tools, skills and experience to make discoveries. 
How is such learning captured or represented? Consider the following quote from a report on the Microscopic Imager in Science (Kerkenhoff et al, 2004)

A typical MI data set includes a stack of three, five, or seven MI images, acquired at 3$\mathrm{mm}$ steps along the MI optical axis with the dust cover open. This acquisition approach helped to ensure optimum focus on targets with relief greater the MI depth of field. The number of images in an MI stack was kept small to minimize the volume of extraneous MI data returned to earth. Most of the MI stacks included at least one image in good focus, but uncertainties in the front Hazcam terrain model resulted in poorly focused MI images in some cases. Color information was sometimes added by acquiring an additional single-frame MI image of the same target at the nominal bestfocus position with the dust cover closed. Some of the MI targets were imaged with a binocular stereo pair of left/right MI stacks or even a mosaic of MI stacks.

This dense paragraph reports tersely the result of hundreds of discoveries of how best to use the instrument, each of which was the result of discussions like the example above. Indeed, as the mission continues with degradation of the rovers' condition and progressive loss of battery power, further learning is taking place about how to use the instruments under extreme conditions, including maneuvers which would not have been even attempted during the initial period of the mission, for fear of damaging the rovers. Without the observation of the actual work of the team, it would be hard to retrieve from this archival record the social activity of the learning which produced it, or even the specifics of each decision.

\section{Learning the Limits of the Models}

A major part of the daily tactical planning process involved scientists individually, in small groups, and in the formal science team meeting of the day, using a software tool called Science Activity Planner (SAP). This tool allows the scientists to specify the observations needed to produce a required observation (and its resulting data product), and provides estimates of the amount of time and power that any given observation would require. It thus allowed scientists to agree on the activities that would be requested on a given day

Thus, for example, if the scientists were considering a drive of a given number of meters, followed by pictures to be taken by specified cameras, SAP could show an estimate of the time it would take to drive to the spot, as well as the amount of power available after the drive. However, these estimates were quite rough. Over time, scientists learned that they could produce plans with power requirements well over the $100 \%$ available power level indicated by SAP

Similarly, odometer figures used for rover location were quite inaccurate, and required compensatory calculations. For example, in a discussion about the strategy for the coming sol, a scientist reminded the group of previous problems with odometry which might compromise the eurrent plan:

"We may have a situation like we did with Faux Trench that our pointing is compromised by the terrain in front. We drove away from Faux Trench with the hope of turning around and shooting it. And we missed wildly because the odometry apparently was wildly wrong. And we may be in a condition like that again. So my point here is that MiniTES on those rocks will probably be a bit dicier than it has been in previous times." 
This example shows the group in the process of learning what activities are possible: a proposed plan is critiqued because the requested images require knowing the precise location of the rover, not possible given the inaccuracy of odometry in the soil of the particular location. Some of the problem of calibrating the odometry were discussed in the first publications of mission results. A discussion of how the exact path of the rover was determined includes the note: "Results indicate that the total length of the rover traverse during the 90 -sol primary mission was $637 \mathrm{~m}$ as measured by wheel odometry, $506 \mathrm{~m}$ as assessied from formal localization performed for the locations where image data were acquired" ((Squyres and al., 2004), p. 205.)

In general, experience allowed the scientists to move from the more conservative estimates provided by the models to increasingly generous estimates in their planning. This kind of learning, however, raises a real question for knowledge management. We do not know whether the SAP software will be carried over for use in future rover missions. If it is, we must ask what mechanisms should be in place to collect the learning about the accuracy of the model's predictions, so that the next version can benefit from this learning. The issue is even more difficult if another modeling program is developed: how can knowledge of the rover's actual behavior under various conditions be preserved for incorporation into the next generration of models?

\section{Strategic Planning}

Oneimportant development, which made the mission so fruitful, was that the science and engineering teams learned to do long term strategic planning, rather than planning one sol at a time. Obviously, the mission had overall strategic goals, both scientific goals ("follow the water"), and mission goals (90 sols of operation, 600 meters driven, etc.). However, the challenge for the team was to bridge between these very high level goals and the intense time pressure of daily operations. Initially, during the field tests on Earth, which served as training both in how to run the rover and how to perform mission operations, scientists tended to plan one sol at a time. However, as they grew more familiar with the planning process, even in the field tests and certainly during the actual mission, they became increasingly adroit at producing multi-sol and even multi-week plans which included complex branching possibilities depending on what was discovered each sol. Strategic planning involved learning how to negotiate a number of tensions: the requirements of different science theme groups, the "bird in the hand' problem", and the development of strategies for producing multi-sol plans with software designed for single-sol planning.

Competition and collaboration among theme groups: The initial model during the field tests was that the different theme groups would work separately to develop their desired plan for the next sol and then would negotiate the final plan during meetings of all the groups. However, as the theme groups grew more familiar with the instruments and the process, they began informal collaborations immediately, working both with intellectually adjoining groups, and with the long term planning group, which served as the custodian of strategic aims. This allowed for the early development of branching plans that ensured that the differing data requirements of the Theme Groups would be obtained within several sols, if not in a given sol.

Additionally, an informal process of monitoring fairness developed. Members of all the Theme Groups noticed situations in which the observations requested by a given Theme Group had not been accepted for several sols, and attempted to ensure that those observations 
were made within a reasonable time. (This was a particular issue for the Atmosphere Theme Group, since its desired observations of sky or horizon were not as dependent on specific locations, and hence on performance on a particular sol, as were those of other theme groups, which required observations of specific rocks or soils.) Interestingly, there were occasions when members of one Theme Group pointed out that another group's requested observations had been postponed for many sols, and that fairness required their requests be given priority.

"Bird in the hand problem": There was a constant tension for scientists between taking as many observations as possible of the location they were in, as opposed to moving on to more obviously interesting locations. The problem, obviously, was that while some locations were more likely to be more geologically significant than others, so little is known about Mars that every location has its charms and possibilities. The tension was heightened by the fact that the tovers had a limited but unknown duration of operation. This was, of course, more of an issue early in the mission, when everything was new It became the task of the chair of the science team (a position which rotated daily or nearly daily) to arbitrate these arguments.

Strategies for multi-sol planning: One problem which the team faced was that the computer tools for science activity planning were designed to handle only a single sol. It was not possible to automatically carry over requested observations to the next sol: such bookkeeping was handled by human memory The ability to handle multi-sol planning, as well as the tension between strategic and tactical aims (the "bird in the hand problem", was assisted by several developments. One was Sol Tree, a representation of alternative multi-sol plans developed by the Long Term Planning Group, and usually publicly visible on the large monitor of a collaborative work tool called the MERBoard. It was extensively used by the scientists in their planning, but did not connect to the representations provided by the Science Activity Planner

Another way of handling the complexities of planning was the development of standard strategies and names for them. This is a well known cognitive strategy chunking - grouping objects or actions into a newly defined entity, and lexicalization - development of new names for these entities. An enormous amount of learning is encoded in these names, and provide a likely mechanism for the preservation of this knowledge across missions. Some examples follow

At the highest level are names describing multi-sol strategies which have become conventionalized. For example, late in the mission, the team developed a "lily pad strategy" a plan for moving the rover from one to another north-facing location in order to take advantage of ayailable solar power, as the advent of the martian winter brought shorter daylight periods, with the Sun in the north.

At the next level down are names for sol types. For example, the 'touch-and-go' sol, which was featured in description of the mission in the first special issue of Science devoted to Spirit at Gusev Crater. (Rowan, 2004) "Spirit drove about 600 meters from the Columbia Memorial Station to the rim of Bonneville Crater Along the way, the rover performed quick analyses, called touch-and-go operations, in which the instruments on the arm touched and analyzed a feature but no brushing or grinding was done. Exploration has changed since the era of nautical exploration by large sailing vessels, when 'touch and go' is thought to have originated to describe a ship's keel touching the seafloor briefly but not getting stuck." 
At al lower level is the combination of several activities, which may be performed during a single sol. These include the 'drive-by' - driving past a specific target and photographing it, the 'scoot and shoot' - a series of drive-bys, a 'scratch and sniff' - drilling into a rock and then examining it with the rover's other instruments, the 'stutter stop' - stopping a drive one meter before the planned end, taking images, and then continuing to the destination. The team also developed terms for types of images, types of features, data downlink issues, and standard strategies for using the science planning software.

\section{Learning How to Learn}

All of these are examples not only of learning the specific technical strategies and solutions, but àlso of learning how to learn as a team, under strict constraints of time, available power, transmissible data volume, and negotiated group demands and strategies. The examples cited here show the learning happening in small groups, taking advantage of the co-presence of team members. That is, the experts needed for consultation were either present, or available by phone (since presumably the team members working on a given rover were operating on the sleep schedule of that rover's time zone.) Team members thus can know who the experts are on particular instruments, software, etc., and can bring them in smoothly to contribute their expertise. Additionally, members of the science and engineering teams moved between rovers, depending on personal schedule issues and scientific interests. Such movement allowed for learning to be transmitted almost immediately from one rover team to another.

This poses an important question for the design of mission operations for future missions. For example, the MSL '09 rover mission has an expected duration of three years, which clearly precludes operation on Mars time, and which requires that participants from institutions other than JPL be able to work at their home institutions. Planning for this kind of learning, and for its dissemination across multiple institutions, nations, and continents requires first recognizing the yalue of these informal, on-the-fly collaborations, and then working seriously on the design of technology for remote collaboration that can allow for analogues or substitutes.

\section{Preśerving (OR Losing) Learning}

Thus far, this paper has attempted to sketch some of the many types of learning which happen as an unremarked part of the daily work of a mission. Obviously, the work of the MER science and engineering teams is anything but routine: team members are creatively performing new and startlingly complex and successful activities in an unexplored terrain on another planet. Certain examples of the team's learning were obvious to the entire world: early loss of data from the rover due to a software problem with flash memory and its successful repair was the stuff of news headlines and ongoing drama (http:/llis.nasa.gov/llis/plls/index.html). But most of the learning discussed here is not dramatic. Rather it is daily, ongoing, incremental. The question for knowledge management is how to recognize this as learning, and ensure that it is preserved.

If knowledge management begins to view knowledge as the product of learning, it is necessary to ask: 'Who learns?' Certainly, individuals learn, and as they move from project to project or mission to mission, there is a good chance that their learning will be available on the mext project. Individuals also train and mentor their colleagues and juniors, providing another way that individual learning is transmitted.

But what would it mean for an institution to learn? Whether it is legitimate to use the terms "learning" and "remembering for institutions has been the subject of a fair amount of debate 
(Walsh and Ungson, 1991). Certainly they make efforts to preserve aspects of what has happened in the past, to find and retrieve some of these representations of the past, and to use them in the present to influence the future. This is done in a variety of ways, ranging from strategies relying on individual human memory and transmission from human to human, through archival and computer storage of documents and data, to organizational policies and procedures and even physical infrastructure. This list is similar to the "bins" of memory proposed in (Walsh and Ungson, 1991): individuals; culture; transformations, which includes training of recruits, standard operating procedures, budgeting, marketing, administrative systems, etc., structures, which include both individual roles within an organization and "institutionalized myths that are sustained and legitimized by members of an organization" ( $p$. 66); and ecology physical structure and design.

Institutions learn by retaining people who have learned, and by engaging them to train others. In a weak sense, it could be argued that an institution has learned by collecting relevant data and making it available as knowledge in archives, databases, etc. However, this constitutes actual learning only if the knowledge is both usable and used. In the strongest sense, an institution has learned when its behavior has changed. Learning has occurred when it has been incorporated into the procedures mandated by the institution, into the design decisions the institution makes, and into the ways in which the institution functions internally and with external partners.

Let $\mu$ s now consider strategies for ensuring that learning is preserved as knowledge, at the indivïdual, team, institutional and scientific levels.

\section{Preșerving Individual Learning}

Although this section discusses individual learning, it must be stressed that in fact no learning can be purely individual, with no contribution to or from other people and groups. This may be appear to be a ridiculous claim in the case of a mathematics student sitting alone in a room with a textbook (but how was the textbook produced?) (Brown and Duguid, 2000). In the case of the MER mission, though, the work is so massively collaborative, depending on the contributions of so many different participants, that learning obviously must be collaborative.

At the same time, particular people do learn, and certain types of learning are carried only by people, with little or no contribution from documentation. There are a number of ways for institutions to preserve this learning: career track planning, mentoring, succession planning. etc..

The career tracks of individuals can make a large difference in whether their learning is preserved for the larger institution. Presumably many of the JPL employees who worked on the MER mission will move on to later Mars missions, thus ensuring that what they have learned is available for these missions. Opportunities for mentoring were present during the MER mission in a number of ways. Senior team members, who had worked on Viking and Pathifinder, NASA's previous Mars lander missions, were part of the team, many of them in roles that gave them responsibility for the strategic planning of the mission. Many of the younger science team members were graduate students of the academic co-investigators: again, a form of mentoring built into the structure of graduate education. They will be well positioned to become investigators for future missions. However, in retrospective assessments of the mission, one question worth asking would be whether there were sufficient 
opportunities and time for effective mentoring to take place at every level of the team struçture.

Some individuals take steps to preserve their individual learning by publishing accounts of their experiences (Mishkin, 2003), (Shirley, 1998), (Sqyres, 2005). However this is a strategy that appears to be available only to fairly high level scientists and managers, and usually for accounts of successful missions.

\section{Preserving Group Learning}

One of the most important types of learning that happened during the mission was that the many people on the MER team, coming from many different institutions, disciplines, and levels of experience, learned to work together under difficult conditions as an extraordinarily effective team. Obviously, the entire team will not work together on the next major Mars mission. However, some key people certainly will participate in this mission and will contribute some of this learning about effective teaming.

A particularly impressive example of this type of learning is the way the various Science Theme Groups and engineering groups learned to work together, allowing the planning processs to occur earlier and earlier in the sol. A question for a retrospective assessment of the mission would be to determine how well the various groups understood the work of other groups and the constraints on it, particularly groups which worked at different times in the cycle, and so did not have the opportunity to learn through face-to-face communications.

The work of learning how to operate on Mars time provides a very detailed example of group learining. Veterans of the Mars Pathfinder mission recalled the difficulties of working on Mars time, and asked Human Factors experts at NASA Ames Research Center for assistance in mitigating the difficulties. A team began with a survey of Pathfinder personnel to determine what factors of Mars time operations were most difficult for them. Schedules, housing facilities, and policies were devised to make this difficult operation as easy as possible. This is learning deep down in the details. Mundane but critical examples include providing shortterm rental apartments for visiting scientists located in quiet neighborhoods near all-night markets, convincing the rental company to provide adequate blackout curtains to allow sleep during the day, providing on-site rooms with cots for "power naps", developing a policy for reimbursing taxi expenses for team members who were too fatigued to drive home safely

\section{Preserving Institutional Learning}

Institutions learn when their members have learned. Thus, developing effective training is an important part of any institutional learning strategy. However, in a more formal sense, institutions have learned when they have developed policies, procedures and practices which incorporate the learning developed by individuals and groups. This is a method which puts the responsibility for incorporating learning on the institution directly As shown by the Columbia Accident Investigation Board Report (Gehman et al, 2003), it is not enough to mandate procedures. It is also necessary to have continued organizational attention to ensure that these procedures are followed, and that they can be followed. The existence of conventionalized workarounds to institutional policies is not, as management might be tempted to think, a testimony to human perversity Rather $r_{p}$ it suggests that the institutional procedures themselves may be impeding the workflow required, or that additional, unacknowledged environmental factors, such as production pressures, may make procedures 
difficult or impossible to follow. Additionally, the rapid tempo of a mission like MER suggests a need for attention to the meta-process for producing procedures. Is it possible to inclide new learning into mandated processes during the mission, or does the time required for validation process for processes make this impossible?

\section{Scientific and Public Learning}

It is aliso important to note that knowledge produced by such a mission travels far beyond the people and the institutions which carry them out. Obviously, the MER mission has already resulted in scientific publications, and the data of the mission will be a source of scientific analyses and publications for decades to come. The mission was unusual in choosing to make its raw data publicly available on its website as soon as possible. It also made determined efforts at reaching the general public, with scientists working in a sustained way with press officers and science education experts to get new, engaging information out rapidly and contịnuously

\section{How Best To Preserve KNoWledge}

Let us conclude by considering how to implement strategies and tools for the preservation of knowledge at all these levels. First, this paper has argued that knowledge management should begin with an understanding that knowledge is the product of learning, individual and collective. Such a view provides a very different perspective for knowledge management than the common assumption that the beginning point for knowledge management is data, which is turned into interpreted information, and finally into usable knowledge. Taking this view suggests an emphasis on identifying where learning is happening, and determining the best ways to support and extend it within an institution, as well as preserving it for future uses.

\section{Lesșons Learned Activities}

A common strategy to achieve institutional learning is to develop Lessons Learned databases for particular missions or projects. This is often done by setting up a Lessons Learned database to which project participants are requested or required to contribute relevant lessons, which are then validated before entry into the official database. At NASA, these Lessons Leained are available both publicly and internally (llis.nasa.gov). In general, a search of lessons for both Pathfinder and MER missions shows a predominance of small scale technical issues. Additionally, the format of the Lessons Learned form requires a description of a * driving event, a description of the lesson learned, and recommendations. This format encourages recording problems rather than positive learning. Further, the form is short, which precludes a detailed description of the kinds of learning and ways of learning described above.

In addititon to the individual process of gathering Lessons Learned described above, there are alsolLessons Learned workshops. These vary widely in format, and the variations make a great difference in the probable effectiveness of the product. The optimal arrangement is to have the activity as part of a scheduled post-mission review, and ensure that the participants include not only mission scientists and engineers, but also institutional decision makers who can derive immediate action items, and junior level employees, who can use the event as an opportunity to learn in preparation for later stages on their career tracks. Using a workshop as a way to populate a database is much less effective, although probably still better than having individuals fill out in the privacy of their own offices, since at least some discussion, argument and cross-learning is possible. 
There have been a number of efforts at NASA to extract and record knowledge from senior or retiring experts. This is a particular issue for missions which may take generations to complete. These activities usually take the form of guided interviews, producing video tapes, raw transcripts, or edited versions of conversations. These efforts share the problem of all oral history projects: the difficulty of effective indexing and formatting. There has been little research on the reuse of such products. Without a great deal of work after the knowledge capture phase, what is produced is raw data, in a form likely to be unusable for anyone who must come up to speed fast in a particular technical area. A number of technological solutions are being attempted in the area of automatic indexing of video records, and these may alleviate some problems. However, thus far, research has shown that the most successful capture efforts of oral lessons learned involve a great deal of skilled writing and editing to make them useable (Linde, 2001). Further, it appears that it does not work to try to capture knowledge first, and figure out how it will be used later Rather, it is necessary from the beginning to design knowledge capture for its potential use and audience.

A recent way to capture knowledge is the development of web-based, interactive sites for posting and discussing lessons learned rapidly. One of the more commonly referenced one is the Eureka system, developed at Xerox PARC to allow a community of copier technicians to share and comment on tips for fixing equipment in the field (Bobrow and Whelan 2002). The war in Iraq has shown that the Center for Army Lessons Learned, often cited as a model of lessons learned production, is too slow to keep up with rapid unexpected developments. Field officers have started web sites for sharing information as diverse as tips on field tactics, understanding Iraqi culture, dealing with suicidal soldiers, and even types of candy that don't melt in desert heat. Initially unofficial, these sites have been migrated to official Army websites (Baum, 2005).

\section{Preparing to Capture Future Learning}

In addition to refining methods for producing Lessons Learned databases, and capturing departing expertise, it is important for the field of knowledge management to develop other ways to accomplish knowledge capture as well. One suggestion would be for every major project to have a learning office or a learning officer, whose job it is to record successful learning as it happens. The MER mission did have a position called "documentarian" whose job was to record the events of each day However, this position tended to be filled by graduate students, whose lack of experience led them to record tactical rather than strategic decisions. Someone holding such a position should be able to recognize and document positive learnings as they occur

In particular, as NASA prepares for the multi-decade, multi-generational exploration of the Moon and Mars, it is necessary to make the best use and record of the learning that happens along the way Let us reflect on what we might want to learn from the Apollo lunar landing missions. While a return to the moon will use very different technologies and procedures, there is still much that was learned during those missions that is still valid and important 35 years later

First, we should distinguish between fields in which there are major changes in the technology, for example, software design, versus those in, which there is incremental progress, such as thermal protection of spacecraft. We would hardly want to remember or use the software used for the Apollo 11 mission in 1969. 


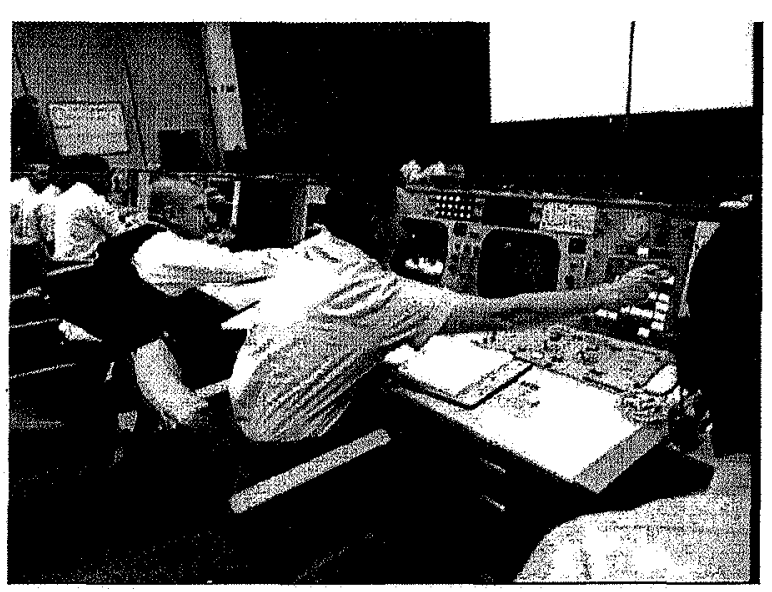

Flight Director's console during the launch of the Apollo 11 lunar landing mission.

In contrast, the root causes of some of the large scale problems of past missions involve management, legal and political pressures which are still in force, and which might provide very! valuable knowledge for new missions. Finally there are constants. everything that we have learned about how to operate under lunar gravity is still relevant for future missions.

Learning from experience requires us both to recognize learning as it happens, and to distinguish those forms of immediate learning that can help us learn in the future.

\section{ACKNOWLEDGEMENTS}

I would like to thank my colleagues on the MER fieldwork team. First, I am grateful to Roxana Wales, who did the intensive, difficult, and usually invisible work of arranging for fieldwork to be possible under the intense conditions of mission operations. I also thank my other team-mates Chin Seah, Valerie Shalin, Zara Mirmalek, and William J. Clancey I am indebted to Cheri Brownton, Lisa Faithorn and Robin Kornman for help at various stages in the preparation of this paper Particular thanks to all the members of the MER mission team who generously allowed us to share this adventure with them. 


\section{REFERENCES}

Baum, D. (2005) What the generals don't know The New Yorker

Bobrow, D. G \& Whalen, J (2002) Community Knowledge Sharing in Practice: The Eureka Story Reflections The SoL Journal.

Brown, J S. \& Duguid, P (2000) The Social Life of Information, Boston, Harvard Business School Press.

Davenport, T H. \& Prusak, L. (1988) Working Knowledge: How Organizations Manage What They Know, Boston, Harvand Business School Press.

Gehman, H. W \& Al, E. (2003) Columbia Accident INvestigation Board Report, NASA and Government Printing Office.

Kerkenhoff, K. E. E. A. (2004) Texture of the Soils and Rocks at Gusev Crater from Spirit's Microscopic Imager Science, 205, 824-6.

Linde, C. (2001) Narrative and Tacit Knowledge Exchange. Journal of Knowledge Management, 5, 160 - 170 .

Maccormack, A. (2004) Management Lessons from Mars. Harvard Business Review, May $2004,18-19$.

Mishkin, A. (2003) Sojourner An Insider's View of the Mars Pathfinder Mission, Berkeley Books.

Rowan, L. (2004) Touch and Go. Science, 205, 793.

Shirley, D. (1998) Managing Martians, Bantam Dell.

Squyres, S. W \& Al., E. (2004) The Spirit Rover's Athena Science Investigation at Gusev Crater, Mars. Science, 205, 793-9.

Sqyires, S. (2005) Roving Mars. Spirit, Opportunity, and the Exploration of the Red Planet, Hyperion.

Wales, R., Shalin, V \& Bass, D. (2005) Requesting Distant Robotic Action: An Ontology of Work, Naming and Action Identification for Planning on the Mars Exploration Rover Mission. Journal of the Association for Information Systems, Special Issue on Ontologies in the Context of Information System)

Walsh, J. P \& Ungson, G. R. (1991) Organizational Memory Academy of Management Review, 16, 57 - 91 